\title{
HIDRÓLISIS Y FASE METANOGÉNICA DE LA DIGESTIÓN ANAEROBIA DE LODOS DE DEPURADORA Y RESIDUOS SÓLIDOS URBANOS
}

\author{
HYDROL YSIS AND METHANOGENIC PHASE OF \\ ANAEROBIC DIGESTION OF SEWAGE SLUDGE AND \\ URBAN SOLID WASTE
}

\section{Tânia Forster Carneiro, Montserrat Pérez}

Centro Andaluz Superior de Estudios Marinos, Espanha. E-mail: tania.forster@uca.es y montserrat.perez@uca.es

\begin{abstract}
RESUMEN
Este trabajo presenta y discute la viabilidad de los procesos anaerobios mesofílicos de lodos de depuradora y residuos sólidos urbanos. El experimento consiste en la puesta en marcha de reactores anaerobios de lodos de depuradora operando en condiciones mesofílicas $\left(35^{\circ} \mathrm{C}\right)$ y semi-continua (TRS 30 y 20 días) y utilizando dos distintas fuentes de inóculo (30\% de inoculación): lodos digeridos y residuos urbanos. Los efectos térmicos, químicos, físicos y biológicos de los tratamientos de los lodos de depuradora y del RSU hasta el metano y el dióxido de carbono son investigados ya sea por separado o conjuntamente. Por último, los parámetros químicos de la hidrólisis y de la fase metanogénica de estos materiales son descritos y comparados con el fin de proporcionar información útil sobre los factores que limitan la digestión anaerobia, así como para sugerir la mejor manera de llevar a cabo el proceso a gran escala.
\end{abstract}

Palabras-claves: Digestión anaerobia. Lodos. Residuos Sólidos Urbanos. Mesofílico/Termofílico.

\begin{abstract}
This paper presents and discusses the feasibility of the mesophilic anaerobic digestion of the sewage sludge and urban solid waste. The experiment consists in the implementation of anaerobic reactors for sewage sludge operating under mesophilic $\left(35^{\circ} \mathrm{C}\right)$ and semi-continuous (TRS 30 and 20 days) and using two different sources of inoculum (30\% inoculation) and digested sludge urban waste. The thermal effects, chemical, physical and biological treatment of sewage sludge and MSW to methane and carbon dioxide were investigated either separately or jointly. Finally, chemical parameters of the hydrolysis and methanogenic phases of these materials are described and compared in order to provide useful information on the factors limiting the anaerobic digestion as well as to suggest the best way to carry out the process on a large scale.
\end{abstract}

Keywords: Anaerobic digestion. Sludge. Urban solid waste. Mesophilic/Thermophilic. 


\section{INTRODUCCIÓN}

En los últimos años, las Administraciones Públicas han venido realizando numerosas actuaciones encaminadas a la mejora de la gestión de los residuos sólidos urbanos (RSU) y los lodos de depuradora (LD) en línea con las directrices y principios emanados de la Unión Europea y contenidos en el Plan Nacional de Residuos Urbanos. En España, la gestión de los lodos generados en las estaciones depuradoras de aguas residuales-EDAR (LD) y de los residuos sólidos urbanos generados en las ciudades están regulados por el vigente Plan Nacional Integrado de Residuos (PNIR) 2007-2015. La adopción de un sistema adecuado de disposición final de los RSU y LD tienen una serie de connotaciones políticas, sociales y económicas, siendo fundamentales los siguientes aspectos: bajo coste de inversión, reciclaje y reutilización de productos, mínima afección al medio ambiente, y escasa necesidad de espacio.

De todas las fuentes de energía renovables, los RSU y los LD son los más importantes en el conjunto de la Unión Europea. En el año 1995, la energía procedente de los residuos sólidos urbanos, ganaderos, agrícolas y forestales, e industriales representó aproximadamente un 55\% (40081 ktep) de las renovables. El biogás generado en la digestión anaerobia de los residuos ganaderos, LD y RSU presenta un gran potencial energético ya que producen gran cuantidad de metano. Así, $1 \mathrm{~m}^{3}$ de biogás con un $60 \%$ de metano tiene un poder calorífico próximo a las 5.500 kcal.

La materia orgánica, como componente mayoritario del residuo debe aprovecharse siempre que sea posible, como recurso biológico. Sin embargo, actualmente, dicho aprovechamiento no siempre es factible, y hay casos en los que la solución más económica es la disposición del residuo en el vertedero (MATA-ALVAREZ et al., 2000). Por todo ello, la solución que permite un aprovechamiento simultáneo y/o individual de los lodos de depuradora y residuos sólidos urbanos está formada por un sistema mixto de reciclaje, compostaje y digestión anaerobia de la fracción orgánica fermentable y revalorización energética (BOLZONELLA et al., 2005).

La digestión anaerobia mesofílica $\left(35^{\circ} \mathrm{C}\right)$ es la opción más ampliamente extendida a escala industrial y a nivel nacional para reducir el volumen del residuo orgánico y obtener energía en forma de gas metano. En este proceso la materia orgánica se transforma en biogás (fundamentalmente metano y dióxido de carbono) de considerable potencial energético (BOUALLAGUI et al., 2005). El biogás se utiliza, generalmente, para el calentamiento de los digestores, en la obtención de energía eléctrica y en el secado de los lodos (PECHARAPLY et al., 2007). Sin embargo, el gobierno español proyecta un aumento del potencial energético a partir de la biomasa. En la práctica supone duplicar la obtención de biogás en digestores a partir de la biomasa con el fin de producir energía y reducir la contaminación del medio ambiente por los residuos sólidos y patógenos, en el caso de los lodos de depuradora (RAIS y AISSA, 1998; KOIVUNENA et al., 2003).

No obstante, los trabajos previos ponen de manifiesto que el proceso anaerobio presenta una relativa complejidad y que se carece de estudios que detallen los procedimientos más adecuados para el arranque de los reactores, según el tipo de residuo (RSU o LD), y otros aspectos tales como el tipo de inóculo más adecuado, las diferentes configuraciones del proceso, porcentaje de sólidos, etc. (BOLZONELLA et al., 2005; FORSTER-CARNEIRO et al., 2008b).

Este aspecto conlleva la necesidad de estudiar las características específicas del arranque y de la fase metanogénica más adecuadas para cada tipo de residuo en particular y en conjunto para optimizar los tiempos de arranque del proceso y la estabilidad y posterior operación del sistema.

El objetivo del presente trabajo es presentar y discutir viabilidad de los procesos de tratamiento mesofílico de lodos de depuradora y residuos sólidos urbanos. Los experimentos se han realizado en reactores a escala de laboratorio y en régimen de operación semi-continuo para 
determinar los máximos rendimientos que pueden alcanzarse, tanto en eliminación de materia orgánica como de producción de metano.

\section{MATERIAL Y MÉTODOS}

\subsection{Procedimiento experimental}

El proceso experimental se diseña para analizar procesos de digestión anaerobia simultáneos y/o individuales con lodos de depuradora y residuos sólidos urbanos, a temperaturas del rango mesofílico $\left(35^{\circ} \mathrm{C}\right)$, y operando en semi-continuo con dos tiempos de retención (TRS 30 y 20 días) así como aplicar dicha información para determinar la eficacia del proceso en la reducción de materia orgánica y producción de metano. El trabajo experimental se desarrollado en dos fases.

1) Digestión anaerobia de lodo utilizando como fuente el lodo digerido mesofílico operando en un reactor de 5L (LD_LD).

2) Digestión anaerobia de lodo utilizando como fuente de inóculo el residuo sólido urbano digerido termofílico operando en reactor de 3L (LD_RSU).

Ambos ensayos fueron realizados en duplicada, no obstante solamente un experimento fue seleccionado como representativo de los resultados.

La caracterización inicial de los reactores y las condiciones de operación impuestas se presentan en la Tabla 1.

Tabla 1 - Caracterización inicial de los reactores y condiciones de operación.

\begin{tabular}{ccccc}
\hline Reactores & $\begin{array}{c}\text { Temperatura } \\
\left(\boldsymbol{C}^{\circ} \mathbf{C}\right)\end{array}$ & $\begin{array}{c}\text { Tiempo de } \\
\text { Retención (días) }\end{array}$ & $\begin{array}{c}\text { Volumen de } \\
\text { Alimentación } \\
(\boldsymbol{m L})\end{array}$ & $\begin{array}{c}\text { Volumen de } \\
\text { Efluente } \\
(\boldsymbol{m L})\end{array}$ \\
\hline LD_LD & $35^{\circ} \mathrm{C}$ & 0 hasta 45 & 150 & 140 \\
& & 46 hasta 100 & 225 & 215 \\
LD_RSU & $35^{\circ} \mathrm{C}$ & 0 hasta 22 & 86,7 & 80 \\
& & 23 hasta 100 & 105 & 100 \\
\hline
\end{tabular}

\subsection{Equipo experimental}

La experimentación se ha llevado a cabo en dos reactores a escala de laboratorio y régimen de operación semicontinuo y discontinuo. Los digestores, ambos del tipo mezcla completa, tienen una capacidad total de tratamiento de $5,0 \mathrm{~L}$ y 3,0 L y han sido utilizados en trabajos previos (FORSTER-CARNEIRO et al., 2007; FORSTER-CARNEIRO et al., 2008a). El reactor se mantiene a $35{ }^{\circ} \mathrm{C}$ y/o $55^{\circ} \mathrm{C}$ gracias a una camisa calefactora, a través de la cual circula agua procedente de un baño termostático. El sistema de agitación está compuesto por un motor de agitación y una pala central; mediante un sistema de aberturas se permite el control del $\mathrm{pH}$ y la toma de muestras del interior del reactor. El sistema posee, además, un sistema de recogida de biogás (mediante bolsas TEDLAR) (Figura 1). 


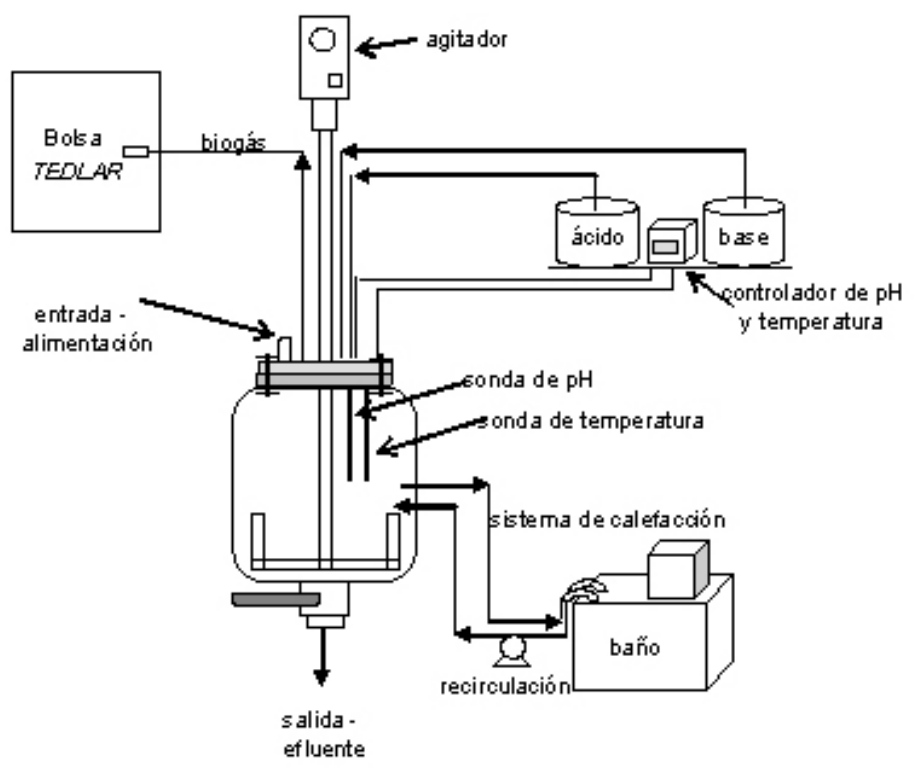

Figura 1. Esquema representativo del digestor tipo tanque agitado (5L).

\subsection{Sustrato e inóculo}

Como sustrato de alimentación de los reactores se utilizan:

1- lodos mixtos (mezcla de primarios y secundarios) procedentes de la EDAR Cádiz-San Fernando (Cádiz) que opera bajo el sistema convencional de lodos activos.

Como fuente de inóculo en los reactores se utilizan:

1- lodos mesofílicos procedentes del efluente de un digestor industrial de la EDAR CádizSan Fernando, que opera en condiciones anaerobias y mesofílica tratando lodos mixtos generados en la planta (TRS de 30 días) y

2- fracción orgánica de residuos sólidos urbanos (FORSU) procedente de un reactor previamente arrancado por el grupo de investigación, que opera en condiciones anaerobias y termofílicas (TRS de 20 días).

\subsection{Análisis microbiológicos}

Las determinaciones de patógenos se realizan en las distintas etapas del proceso, para lodos mixtos de depuradora y lodos digeridos mesofílicos.

Según los métodos recogidos en los Métodos Normalizados para el análisis de aguas potables y residuales (APHA, 1989) y las normas EPA (1992), se utilizam los siguientes métodos de análisis en lodos de depuradora

1) Método filtración en membrana (Medio de cultivo m-FC) para la detección de Coliformes totales y fecales: Este método consiste en filtrar un volumen adecuado de muestra de lodo (dilución 1:100) a través de una membrana estéril de $0,45 \mu \mathrm{m}$. Posteriormente la membrana se pone en contacto con el medio m-FC, que da lugar al crecimiento de colonias azules. Para la detección de Coliformes fecales la incubación se hace en estufa a $44,5^{\circ} \mathrm{C}$ durante 24 horas y el recuento de colonias se hace con arreglo al NMP.

2) Método de detección de Salmonella sp.: Las muestras son diluidas con agua de peptona estéril. A continuación son inoculadas en medio de enriquecimiento RappaportVassiliadis (segunda etapa) a partir del método de tubos múltiples (N. 9260-B) durante 24 horas en incubadora a $40{ }^{\circ} \mathrm{C}$ de temperatura (el cambio de color a verde indica presencia 
de Salmonella sp.). Los tubos positivos de cada hilera permiten identificar el índice $\mathrm{NMP} / 100 \mathrm{~mL}$ en la tabla de índice de NMP del Standard Métodos. Utilizando los datos de índice $\mathrm{NMP} / 100 \mathrm{~mL}$ y el porcentaje de sólidos totales de la muestra se calcula la concentración de Salmonella sp. (NMP/4g ST). Finalmente, se hace el aislamiento de los tubos positivos en placas con Agar XLD durante 24 horas a $37^{\circ} \mathrm{C} \pm 0,2^{\circ} \mathrm{C}$ y se confirman todas las placas positivas a través de un método de confirmación comercial (tercera etapa).

\subsection{Análisis físicos y químicos}

Diariamente se procede a alimentar los digestores con la cantidad de lodo impuesta según el TRS fijado en el sistema. Los parámetros analizados son: $\mathrm{pH}$, Sólidos Totales (ST), Sólidos Volátiles (SV) y materia orgánica (medida como DQO) así como la alcalinidad y el contenido en ácidos grasos volátiles (Acidez Total) mediante cromatografía. El volumen y la composición $\left(\mathrm{H}_{2}\right.$; $\mathrm{O}_{2} ; \mathrm{N}_{2} ; \mathrm{CO}_{2}$ y $\mathrm{CH}_{4}$ ) del biogás se analizan diariamente mediante cromatografía gaseosa. Las determinaciones analíticas se realizan de acuerdo a los métodos estandarizados (APHA, 1989), adaptándolos a residuos de alta carga orgánica y alto contenido en sólidos.

\section{RESULTADOS Y DISCUSIÓN}

En la Tabla 2 se presenta una caracterización inicial del sustrato utilizado (LD) (valores medios de las diversas alimentaciones utilizadas a lo largo del ensayo) y de las fuentes de inóculos (lodo y residuo sólido urbano). En esta tabla se observa que os los lodos mixtos poseen un alto contenido en SV, del orden de 2,5\%, así como un alto contenido de carga orgánica, 3400 $\mathrm{mgDQO} / \mathrm{L}$, típico de un residuo fresco no estabilizado. Al contrario, ambos inóculos, lodo mesofílico procedente de los digestores industriales de la EDAR Cádiz-San Fernando y RSU termofílico poseen menor contenido en SV, del orden del 1,7\% y una carga orgánica entre los 6000 y $10.000 \mathrm{mgDQO} / \mathrm{L}$, respectivamente.

La etapa de arranque y adaptación del digestor se desarrolló hasta alcanzar condiciones estables de operación en condiciones mesofílicas a TRS 30 días simulando el tiempo de retención al que opera el digestor industrial. A partir de estas condiciones, y una vez alcanzada estabilidad a TRS 30 días, se procedió a disminuir el TRS hasta 20 días y a evaluar los principales parámetros de operación. 
Tabla 2 - Composición y caracterización físico-químico iniciales de los reactores.

\begin{tabular}{|c|c|c|c|c|}
\hline \multirow[t]{2}{*}{ Parámetros Analíticos } & \multicolumn{2}{|c|}{$\begin{array}{l}\text { Reactor } \\
L D \_L D\end{array}$} & \multicolumn{2}{|c|}{$\begin{array}{c}\text { Reactor } \\
L D \_R S U\end{array}$} \\
\hline & $\begin{array}{l}\text { Sustrato } \\
\text { Lodo } \\
\text { Mixto } \\
\end{array}$ & $\begin{array}{c}\text { Inóculo } \\
\text { Lodo } \\
\text { Mesofilico }\end{array}$ & $\begin{array}{l}\text { Sustrato } \\
\text { Lodo } \\
\text { Mixto } \\
\end{array}$ & $\begin{array}{c}\text { Inóculo } \\
R S U \\
\text { Termofilico } \\
\end{array}$ \\
\hline Densidad $\left(\mathrm{kg} / \mathrm{m}^{3}\right)$ & 1025,6 & 1054,0 & 1025,6 & 972,0 \\
\hline Materia Orgánica (\%) & 69,4 & 56,2 & 69,4 & 71,4 \\
\hline Humedad (\%) & 96,4 & 96,8 & 96,4 & 97,7 \\
\hline Sólidos (\%) & 3,6 & 3,2 & 3,6 & 2,3 \\
\hline $\mathrm{ST}(\mathrm{g} / \mathrm{kg})$ & 36,0 & 32,0 & 36,0 & 23,3 \\
\hline $\mathrm{SV}(\mathrm{g} / \mathrm{kg})$ & 25,0 & 18,0 & 25,0 & 16,7 \\
\hline STS (g/L) & 31,4 & 26,6 & 31,4 & 17,9 \\
\hline $\operatorname{SVS}(\mathrm{g} / \mathrm{L})$ & 25,8 & 19,0 & 25,8 & 12,8 \\
\hline $\mathrm{pH}$ & 7,3 & 7,7 & 7,3 & 5,1 \\
\hline Alcalinidad (mg/L) & 4030,0 & 3080,0 & 4030,0 & 5864,0 \\
\hline Acidez Total (mg/L) & 851,0 & 1718,0 & 851,0 & 2140,0 \\
\hline $\mathrm{DQO}(\mathrm{mg} / \mathrm{L})$ & 3375,0 & 9582,6 & 3375,0 & 5922,7 \\
\hline Coliformes totales $(\mathrm{NMP} / 100 \mathrm{~mL})$ & $5 \times 10^{4}$ & $2,0 \times 10^{4}$ & $5 \times 10^{4}$ & $1,2 \times 10^{3}$ \\
\hline Salmonella sp. (NMP/100mL) & 10,3 & 0,67 & 10,3 & 1,27 \\
\hline
\end{tabular}

En la Figura 2a se presenta la evolución temporal de materia orgánica en el digestor expresado como sólidos volátiles. Según se observa, en ambos digestores (en semi-discontinuo) la eliminación de SV se mantiene constante hasta el final de la experimentación. En estas condiciones y tras 100 días de experimentación, los digestores LD_LD y LD_RSU mantienen porcentajes de eliminación de SV respecto a los sólidos de la alimentación, superiores al $80 \%$. Resultados similares son observados cuanto la porcentaje de eliminación de materia orgánica expresado como demanda química de oxígeno (DQO) (Figura 2b). Los digestores que operan en semi-discontinuo mantienen un porcentaje de eliminación de la DQO respecto a la DQO de la alimentación del orden de $70-80 \%$. 

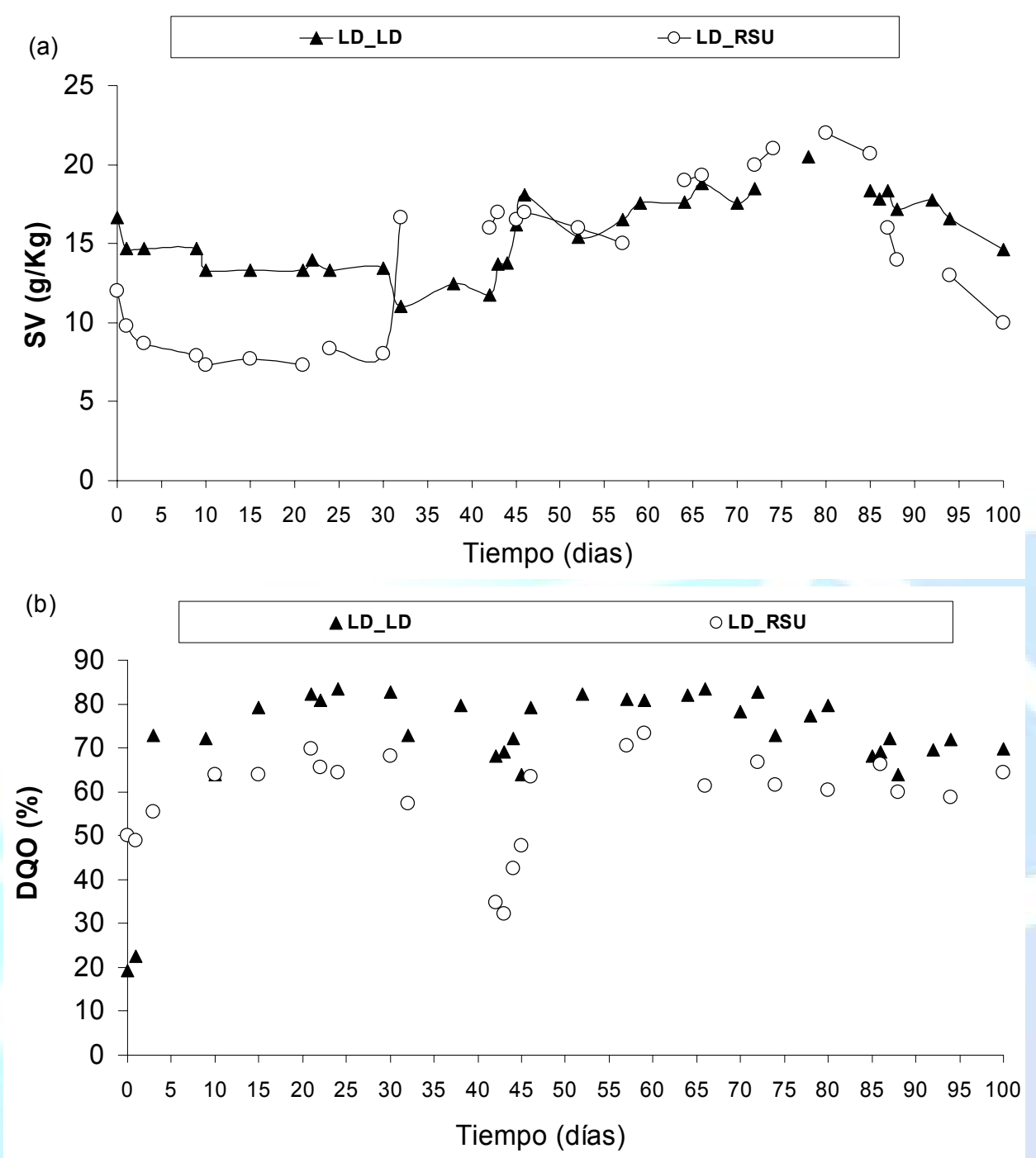

Figura 2. Evolución temporal de materia orgánica en función del tiempo expresado como sólidos volátiles (SV) en (a) y porcentaje de eliminación de materia orgánica en función del tiempo expresado como demanda química de oxígeno (DQO) en (b).

Respecto a los parámetros de control y seguimiento del reactor, se presenta en la Figura 3a la evolución temporal de los niveles de alcalinidad $(\mathrm{mg} / \mathrm{L})$ y en la Figura $3 b$ la evolución temporal de los niveles de acidez total (mg/L).

Se puede observar valores de alcalinidad del efluente de los reactores LD_LD muy estables durante toda la experimentación, manteniéndose en torno del $3500 \mathrm{mg} / \mathrm{L}$. No obstante, en el reactor LD_RSU, que opera en discontinuo, se observa una fase inicial con valores muy altos, $6800 \mathrm{mg} / \mathrm{L}$ en el primero día, y luego un período de estabilidad entre los días 10 y 70 con valores entre 3000 y $4000 \mathrm{mg} / \mathrm{L}$, posiblemente debido a la adaptación del sustrato (lodo mixto) al inóculo de RSU termofílico (Figura 3a).

En la Figura 3b se observa los niveles de acidez total bajos en el entorno de 3500-3000 $\mathrm{mgCaCO} / \mathrm{L}$ en el reactor LD_LD durante casi toda la experimentación, incrementado la acidez del medio en la última etapa del proceso. Al contrario, el reactor LD_RSU presenta valores estables y adecuados a la degradación anaerobia, se puede observar valores del orden de los $2800 \mathrm{mg} / \mathrm{L}$ en los primeros 45 días de ensayo, correspondientes al TRS de 30 días y, a continuación valores 
constantes $(2000 \mathrm{mg} / \mathrm{L})$ hasta el final de la experimentación (TRS de 20 días). Estos resultados indican la presencia de una la fase de arranque e hidrólisis con altos valores de alcalinidad, y niveles moderados en la fase metanogénica. En la Figura 4a se presenta el porcentaje de metano (\%) y en la Figura $4 \mathrm{~b}$ el acumulo de biogás en función del tiempo.

En el reactor LD_LD se observa valores crecientes de porcentaje de metano y producción de biogás en toda la experimentación. A partir del día 45 (TRS: 20 días) las producciones de biogás y metano se presentan estables hasta el final del experimento, con una media de $60 \%$ de metano (Figura 4a) y un acumulo de 260 L de biogás, tras 100 días de ensayo (Figura 4b).

En el reactor LD_RSU se observa en los primeros 22 días de ensayo altos porcentajes de eliminación de la materia orgánica (60-70\%) y metano en el biogás (40-60\%) lo que supone una importante ventaja para su aplicación a escala industrial (TRS: 30 días). A continuación el porcentaje de metano en el biogás se estabiliza en el entorno del $40 \%$ hasta el final de la experimentación pero el acumulo de biogás no supera los 50 Litros (TRS: 20 días) (Figura 4).

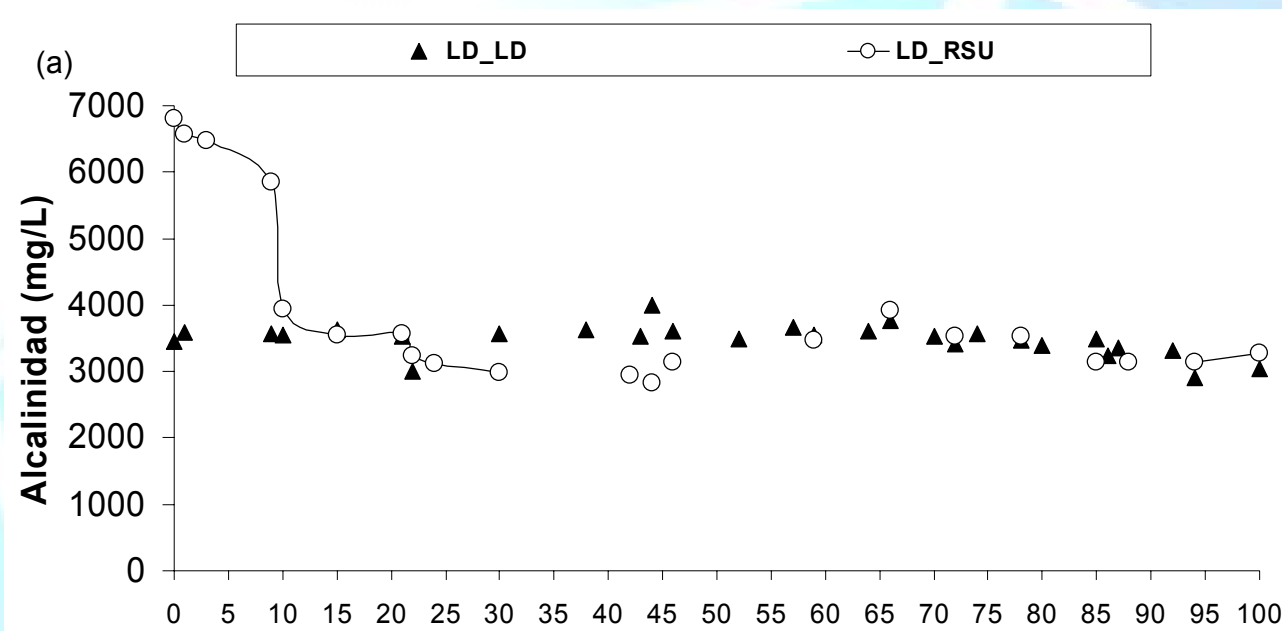

Tiempo (dias)

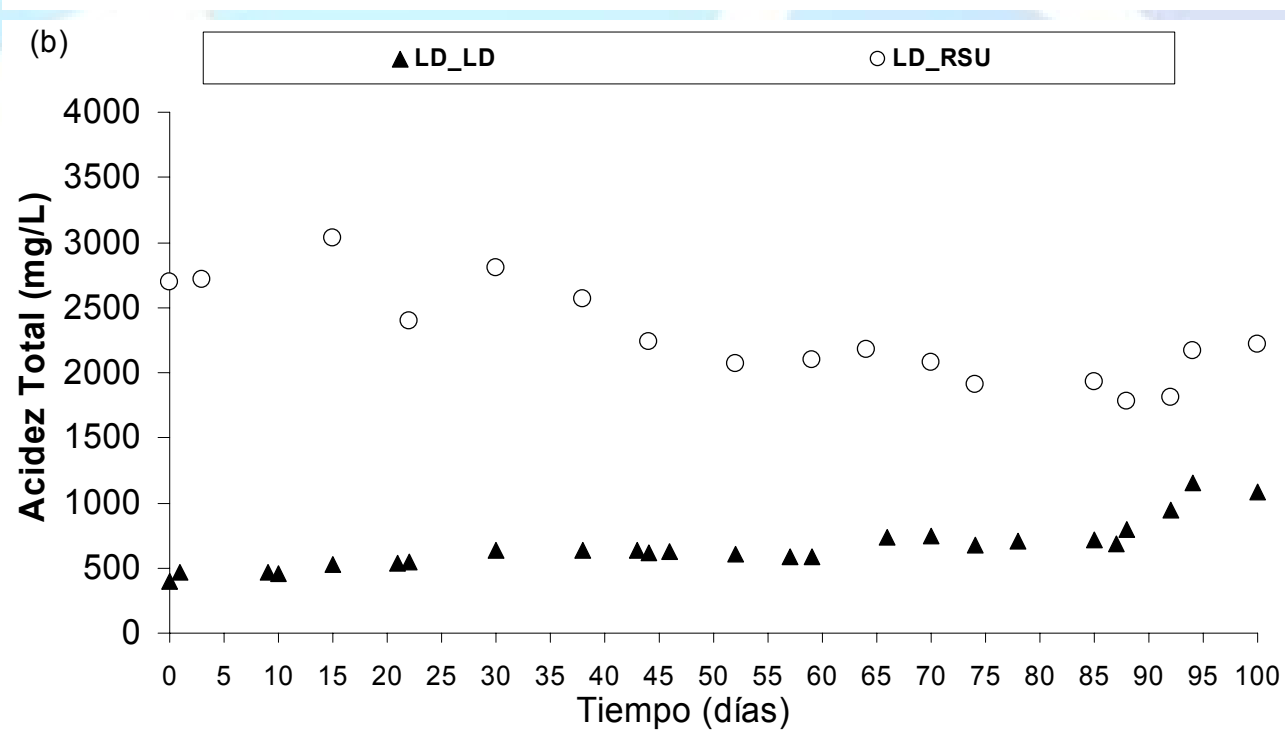

Figura 3. Evolución temporal de alcalinidad ( $\mathrm{mg} / \mathrm{L}$ ) en (a) y de la acidez total (mg/L) en (b) en función del tiempo. 

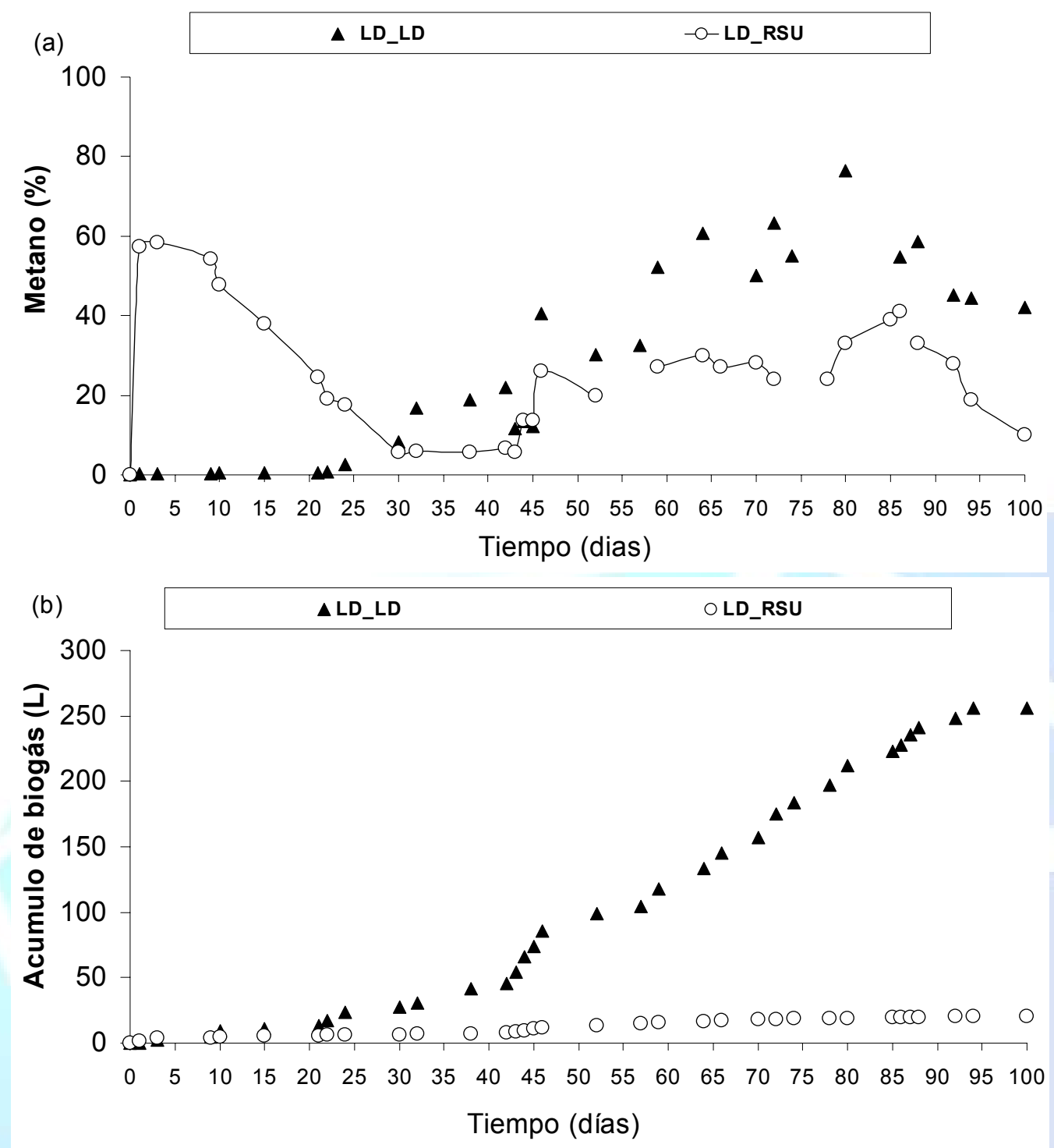

Figura 4. Evolución temporal del porcentaje de metano en (a) y del acumulo de biogás (L) en (b) en función del tiempo.

\section{CONCLUSIONES}

Considerando el objetivo previsto y teniendo en cuenta los resultados obtenidos en este trabajo se pueden extraer las siguientes conclusiones sobre los ensayos de puesta en marcha de reactores anaerobios de lodos de depuradora operando en condiciones mesofílicas $\left(35^{\circ} \mathrm{C}\right)$ y semicontinua (TRS 30 y 20 días) y utilizando dos distintas fuentes de inóculo ( $30 \%$ de inoculación):

1- El reactor LD_LD tarda 45 días en alcanzar la estabilidad del sistema en condiciones de TRS de 30 días. No obstante, una vez alcanzado la estabilidad del sistema se observa, valores crecientes del porcentaje de metano (media de 60\%) y producción de biogás (acumulo de $260 \mathrm{~L}$ de biogás) hasta el final de la experimentación (TRS 20 días).

2- El reactor LD_RSU obtiene un arranque más rápido del proceso, presentado en los primeros 22 días de ensayo altos porcentajes de eliminación de la materia orgánica (60-70\%) y metano en el biogás (40-60\%) lo que supone una importante ventaja para su aplicación a escala 
industrial. No obstante, los porcentajes de metano y la producción de biogás fueron significativamente menores que el reactor LD_LD en condiciones de TRS de 20 días.

Por todo ello, las diferentes fuentes de inóculo presentan diferentes evoluciones en los parámetros químicos indicando una diferenciación temporal espontánea de las diferentes etapas del proceso.

\section{AGRADECIMIENTOS}

El presente estudio forma parte del desarrollo del plan experimental de los Proyectos de Investigación 292/2006/3-4.2 y A130/2007/4-3.2, "Optimización de la digestión anaerobia en fase secuencial de temperatura de lodos de EDAR", financiados por la Secretaría General para la Prevención de la Contaminación y el Cambio Climático del Ministerio de Medio Ambiente.

\section{REFERENCIAS}

APHA - AMERICAN PUBLIC HEALTH ASSOCIATION Standard methods for the examination of water and wastewater, AWWWA, WPCF. Washington, DC. $17^{\text {th }}$ (ed.) 1989.

BONZONELLA, D.; PAVAN, P.; MACE, S.; CECCHI, F. Dry Anaerobic digestion of differently sorted organic municipal solid waste: a full scale experience. In: Fourth International Symposium of Anaerobic digestion of Solid Waste, Copenhagen, Denmark, v. 1, p. 85-92, 2005.

BOUALLAGUI, H.; TOUHAMI, Y.; BENCHEIKH, R.; HAMDI, M. Bioreactor performance in anaerobic digestion of fruit and vegetable wastes. Process Biochemistry, v. 40, p. 989-995, 2005.

DE LA RUBIA, M.A.; ROMERO, L.I.; SALES, PEREZ, D.M. Temperature conversion (mesophilic to thermophilic) in anaerobic sludge digestion. Aiche Journal, v. 51, n. 9, p. 25812586, 2005.

EPA - Environmental Protection Agency. Standards for the use or disposal of sewage sludge (40 Code of Federal Regulations Part 503). Washington D.C. 1992.

FORSTER-CARNEIRO, T.; PÉREZ GARCÍA, M.; ROMERO GARCÍA, L.I.; SALES, D. Dry thermophilic digestion of organic fraction of the municipal solid waste: focusing on the inoculum sources. Bioresource Technology, v. 98, n. 17, p. 3195-3203, 2007.

FORSTER-CARNEIRO, T., PÉREZ GARCÍA, M., ROMERO GARCÍA, L.I. Thermophilic anaerobic digestion of source-sorted organic fraction of municipal solid waste. Bioresource Technology, v. 99, n. 15, p. 6763-6770, 2008a.

FORSTER-CARNEIRO, T., PÉREZ GARCÍA, M., ROMERO GARCÍA,L.I. Influence of total solid and inoculum contents on performance of anaerobic reactors treating food waste. Bioresource Technology, v. 99, n. 15, p. 6994-7002, 2008b.

KOIVUNENA, J., SIITONENB, A., HEINONEN-TANSKIA, H. Elimination of enteric bacteria in biological-chemical wastewater treatment and tertiary filtration units. Water Research, v. 37, p. 690-698, 2003. 
MATA-ALVAREZ, J.; MACE', S.; LLABRES, P. Anaerobic of organic solids wastes. An overview of research achievements and perspectives. Bioresource Technology, v. 74, p. 3-16, 2000.

PECHARAPLY, A.; PARKPIAN, P.; ANNACHHATRE, A.P.; JUGSUJINDA, A. Influence of anaerobic co-digestion of sewage and brewery sludges on biogas production and sludge quality. Journal of Environmental Science and Health and Environmental Engineering, v. 42, n. 7, p. 911-923, 2007.

PNIR - Plan nacional integrado de residuos (PNIR). Anexo 5: II Plan nacional de lodos de depuradoras de aguas residuales - EDAR II PNLD. 2007-2015.

RAIS, T.M.; AISSA, R.B. Contamination bactérienne des bous dépuration utilises à des fins agricoles en Tunisie. Journal Europeen d'Hydrologie, 28:339-354. 1998. 